\title{
Assessment of probiotic effects on colorectal surgery complications: A double blinded, randomized clinical trial
}

\author{
Farzad Kakaei ${ }^{1}$, Mohammadreza Shahrasbi ${ }^{2}$, Touraj Asvadi Kermani ${ }^{3},{ }^{*}$, Sahar Taheri ${ }^{4}$, Kowsar Tarvirdizade $^{5}$
}

${ }^{1}$ Associate Professor of Surgery and Transplantation, Department of General and Vascular Surgery, Tabriz University of Medical Science, Tabriz, Iran

${ }^{2}$ Asisstant of General and Vascular Surgery, Department of General and Vascular Surgery, Tabriz University of Medical Science, Tabriz, Iran

${ }^{3}$ Asisstant Professor of General and Vascular Surgery, Fellowship of Trauma, Department of General and Vascular Surgery, Tabriz University of Medical Science, Tabriz, Iran

${ }^{4}$ Asisstant of Gynecology and Obstetrics, Department of Gynecology and Obstetrics, Alzahra Hospital, Tabriz University of Medical Science, Tabriz, Iran

${ }^{5}$ General Practitioner, Faculty of Medicine, Tabriz University of Medical Science, Tabriz, Iran

\section{Correspondence}

Touraj Asvadi Kermani, Asisstant Professor of General and Vascular Surgery, Fellowship of Trauma,

Department of General and Vascular Surgery, Tabriz University of Medical Science, Tabriz, Iran

Email: tooraj_asvadi2005@yahoo.com History

- Received: Feb 04, 2019

- Accepted: Feb 17, 2019

- Published: Mar 31, 2019

DOI :

https://doi.org/10.15419/bmrat.v6i3.529

\section{Check for updates}

Copyright

(๑) Biomedpress. This is an openaccess article distributed under the terms of the Creative Commons Attribution 4.0 International license.

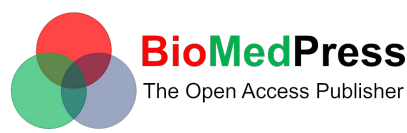

\section{ABSTRACT}

Background: Probiotics are living bacteria, which can be used as a food supplement to produce inhibitory agents and compete with pathogens in the guts. Nowadays, because of the easy accessibility and misuse of antibiotics, probiotic usage is increasing. The goal of our study is to assess the effects of probiotics on colorectal surgery complications. Methods: The enrolled cases were selected from colorectal surgery candidate patients, who referred to Sina and Imam Reza Hospitals of Tabriz University from April of 2016 to April of 2017. They were divided into two groups of study and control. For the study group, 7 days before surgery, one capsule of probiotics (Familact) was administrated every night, while the control group was given capsules of placebo. Results: Although the infective complications, mortality ratio and duration of hospitalization in the study group were lower than the control group, these differences were not statistically significant. Discussion: Our study confirmed that preoperative probiotic consumption in colorectal surgery had not affected complications or mortality of surgery and this finding is in disagreement with some previous studies. Perhaps, other strains of bacteria or different dosage, duration and rout of administration can have positive effects on these complications. Conclusion: This study showed that there was no correlation between probiotic consumption preoperatively and decreased complications or mortality after colorectal surgery.

Key words: Colorectal surgery, Complications, Probiotics

\section{INTRODUCTION}

Probiotics are living bacteria that can be used as food supplementary to produce inhibitory agents to compete with pathogens in the guts. They can stimulate and regulate the immune system and improve the balance of intestinal microbes ${ }^{1-3}$.

Recent studies showed that probiotics can reduce the absorption of cholesterol and decrease the risk of colorectal cancer ${ }^{4}$. Probiotics can also help improving diseases such as diarrhea, constipation, allergies, inflammatory bowel disease, irritative bowel syndrome, peptic ulcer and lactose intolerance, and can prevent autoimmune diseases and hypertension ${ }^{5-7}$.

The combination of probiotics and prebiotics (food ingredients that induce the growth or activity of beneficial microorganisms) is known as "synbiotics" ${ }^{8}$. Synbiotics help to improve the resistance of the gastrointestinal tracts to infected diseases by improving the immune system and antimicrobial activities ${ }^{9}$.

Probiotics colonize in the gastrointestinal tract and oropharynx. They prevent the changes of normal flora into pathogens and have preventive effects on hospital pneumonia ${ }^{8,10,11}$.

Nowadays because of the easy accessibility and misuse of antibiotics, their usage is increasing, which leads to growing resistance of the microorganisms ${ }^{12}$. Recent studies showed that preoperative administration of probiotics in patients with colorectal cancer can reduce infective complications after surgery ${ }^{13-15}$. The goal of our study is to assess the effects of probiotics on the colorectal surgery complications.

\section{METHODS}

This is a randomized clinical trial and a prospective study. The enrolled cases were selected from colorectal surgery candidate patients, who referred to Sina and Imam Reza Hospitals of Tabriz University of Medical Sciences, Tabriz, Iran, from April 2016 to April 2017. All 18- to 80-year-old patients with good nutritional status were divided into two parallel groups of study and control.

Exclusion criteria were set for patients with no agreement for participation in study, having intestinal or colon obstruction, consuming anticoagulant agents or any other drugs that prolong duration of hospitalization, having progressive cardiopulmonary diseases or Class IV of ASA (American Society of Anesthesiologists) or chronic renal failure, having a history of chemotherapy, or patients with organ transplantation, cirrhosis, antibiotics usage one week be- 


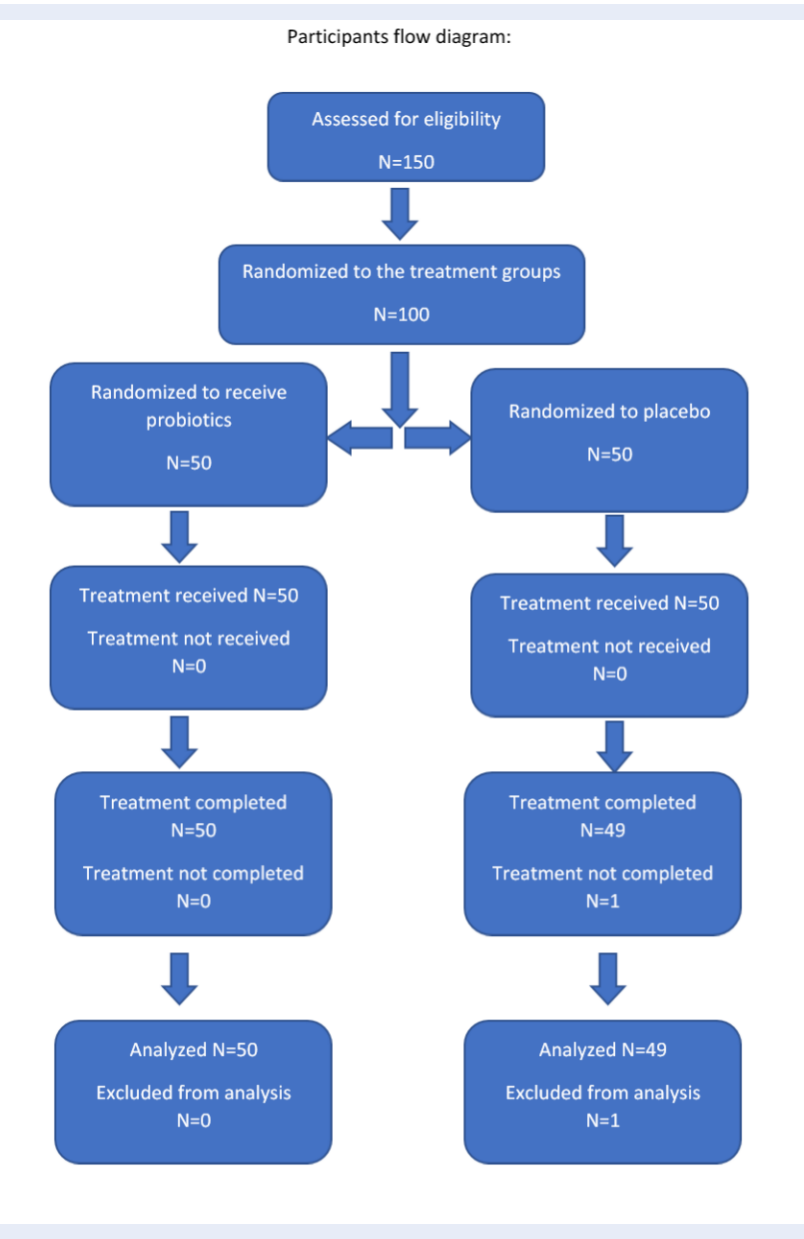

Figure 1: Flow diagram of recruitment and retention of participants in the study: due to death of patient only one patient in control group dropped out.

fore surgery, consuming steroids and being pregnant or lactating.

\section{Randomization and blinding}

We used statistical software in $\boldsymbol{w w w}$.randomizer.org for double blinded randomization. Two equal groups received either probiotics or placebos. Each group consisted of 50 patients and the sample size was determined according to previous studies ${ }^{15}$. Two boxes labelled A (probiotics) and B (placebo) were prepared Figure 1. The patient's nurses were also blinded and received sealed envelopes of letter A or B Patients will be given capsules based on the letters defined on the envelopes. All data was collected by Dr. M.SH., who was also blinded to the types of drugs during 30 days of follow-up.

\section{Procedure}

For the study group, 7 days before surgery, one capsule of probiotics (Familact) was administered every night. Each capsule contains Lactobacillus casei \& Lactobacillus acidophilus $\left(1.75 \times 10^{9} \mathrm{CFU}\right)$, Lactobacillus plantarum $\left(0.5 \times 10^{9} \mathrm{CFU}\right)$, Bifidobacterium breve $\left(1.75 \times 10^{9} \mathrm{CFU}\right)$, Bifidobacterium longum (1.5 $\left.\mathrm{x} 10^{9} \mathrm{CFU}\right)$, and Streptococcus thermophilus, and the control group was given placebo capsules.

For bowel preparation, one day before surgery, four packets of oral polyethilenglycol with two litres of water was administrated. All patients took oral erythromycin $800 \mathrm{mg}$ and metronidazole $500 \mathrm{mg}$ at 13, 14 and 23 oclock. Thirty minutes before operation, cefazolin was infused as prophylactic antibiotics, and if there was no contamination after 48 hours, this step was discontinued; however if there was contamination, antibiotic prescription was changed by attending surgeons. 
After the operation, each patient was given one oral capsule daily when awake. Each patient was given 30 capsules in total.

The control group received similar treatments except that placebo was used instead of probiotics.

\section{Measures}

Variables of the study including gender, age, past medical history, measurement of haemoglobin, incidence of anastomosis leakage, cutaneous or subcutaneous infection on incision line, facia dehiscence, other infections (pneumonia, urinary tract infection, central venous catheter infection and sepsis), duration of hospitalization and mortality ratio were defined.

\section{Statistical Analysis}

All data were analysed by SPSS version of 15 . We used chi-square test for qualitative variables analysis and $t$ test for quantitative variables. P-Values of $\leq 0.05$ were considered reliable.

\section{Ethics}

The study design was approved by Human Research Review and Ethics Committee of Tabriz University of Medical Sciences. All patients were informed about the study, and they had to sign the consent letter for voluntary cooperation. Also, they could leave the study at any time. All personal information were confidential. The only intervention in the treatment was the prescription of Familact capsules. A company of Zist Takhmir produced these capsules as OTC (Over-The-Counter) drugs that had no complications in studies until now. Costs of study were funded by the Vice Chancellor for Research (VCR) of Tabriz University of Medical Sciences.

\section{RESULTS}

There were no significant differences in gender, age, past medical history and haemoglobin levels between two groups (P-value> 0.05) (Table 1).

There were no reports of anastomosis leakage or facia dehiscence from two groups. Three patients (6\%) in the study group and 5 patients (10\%) from control group developed cutaneous and subcutaneous tissues infection on incision line after surgery. However, this difference was not statistically significant ( $\mathrm{P}$-value = 0.46). There was one case of central venous catheter infection in the study group. There was one case of pneumonia that led to respiratory failure and death, and one case of central venous infection in control group, however, there were no significant differences
(P-value $=0.60$ and 0.31$)$ in other infective complications and mortality ratio between two groups. Mean of hospitalization duration of the study group was $5.96 \pm 2.53$ days $(\operatorname{Max}=12$ days, $\operatorname{Min}=3$ days $)$ and of the control group was $6.10 \pm 2.44$ days $(\operatorname{Max}=15$ days, Min $=3$ days), which showed no significant differences $(\mathrm{P}$-value $=0.30)$ Table 2 .

\section{DISCUSSION}

In this study, the effects of probiotic consumption on complications of elective colorectal surgery and its mortality ratio was assessed. The results showed that probiotics had no positive effect on patients after surgery.

McNaught et al. (2002) showed that preoperatively (1 week) oral probiotic (lactobacillus + plantarum 299v) administration in 129 patients with elective abdominal surgery had no positive effects on the growth of beneficial bacteria in guts, nor did it help to improve the resistance of intestinal cells or decrease the postoperative complications and mortality ${ }^{16}$. These results were in agreement with our study.

In the study of Anderson and McNaught et al. (2004), candidate patients for elective abdominal surgery took synbiotics (probiotics such as Lactobacillus acidophilus La5 with prebiotics like oligofructose) for 2 weeks. There was no significant difference between control and study groups, which confirmed our study results ${ }^{17}$.

Most studies in these field showed different results. In the study of Sagawara et al. (2006), patients, who were candidates for hepatectomy with bile duct carcinoma and liver hilum involvement, were divided into two groups of receiving synbiotics or placebo (for two weeks before surgery). The results showed that the administration of synbiotics preoperatively increased lymphocytes and natural killer activities, and decreased the production of IL-6. Also, in the group receiving synbiotics only after surgery, white blood cells count was significantly lower compared to control group, thus, synbiotic administration before surgery could improve the immune system ${ }^{18}$.

Rayes et al. (2007) showed that synbiotics had positive effects on patients. They administrated synbiotics through intestine and found that infection ratio and antibiotic requirements in group treated with lactobacillus probiotics were significantly lower compared to controls ${ }^{19}$.

Liu et al. (2011) in a randomized clinical trial had prescribed oral probiotics to colorectal cancer patients 6 days before and 10 days after colorectal surgery, and 
Table 1: Patient characteristics

\begin{tabular}{|c|c|c|c|}
\hline & $\begin{array}{l}\text { Group received probi- } \\
\text { otics }\end{array}$ & Group received placebo & P-value \\
\hline Age, mean $\pm S D(y)$ & $50.08 \pm 12.64$ & $48.92 \pm 11.23$ & 0.40 \\
\hline Male Gender, n (\%) & $29(58)$ & $26(52)$ & 0.68 \\
\hline Past Medical History, n (\%) & & & 0.71 \\
\hline - No disease & $29(58)$ & $31(62)$ & \\
\hline - Diabetes Mellitus type II & $8(16)$ & $4(8)$ & \\
\hline - Hypertension & $7(14)$ & $11(22)$ & \\
\hline - Heart Disease & $3(6)$ & $2(4)$ & \\
\hline - Familial Colorectal Cancer & $1(2)$ & $1(2)$ & \\
\hline
\end{tabular}

Table 2: Comparing of Colorectal Surgery's Complications in two groups of probiotic consumption and not

\begin{tabular}{llll}
\hline & Probiotic Received & Placebo Received & P-value \\
Anastomosis Leakage & $0 \%$ & $0 \%$ & 1 \\
Fascia Dehiscence & $0 \%$ & $0 \%$ & 1 \\
Infection of Surgical Site & $6 \%$ & $10 \%$ & 0.46 \\
Other Infective Complications & $2 \%$ & $4 \%$ & 0.60 \\
Mean of Hospitalization Duration & $5.96 \pm 2.53$ & $6.10 \pm 2.44$ & 0.30 \\
Mortality & $0 \%$ & $2 \%$ & 0.31 \\
\hline
\end{tabular}

assessed theme effects on bowel activities and postoperative infections. Unlike us, they showed that probiotics could reduce postoperative infections. On the other hand, this study revealed positive effects of probiotics in a diversity of intestinal flora and the excretion of various bacteria in the stool. Therefore, they recommended probiotics as a drug to use before surgery that can increase the resistance of epithelial cells to toxins and inflammative factors ${ }^{15}$.

Liu et al. (2013) examined the effects of probiotics on the levels of serum xenoline and their association with infection rates. In this study, 150 patients of colorectal cancer were divided into two groups of controls and cases and received oral probiotics 6 days before- and 10 days after surgery. The results showed that the probiotics had positive effects on the significant reduction of serum xenoline and on reducing intestinal permeability, which led to decreased postoperative infections and antibiotic requirement ${ }^{13}$.

Liu et al. in another study (2015) concluded that probiotics reduced postoperative infections in patients with liver metastasis ${ }^{20}$.

Three systematic reviews (2017) and one metaanalysis (2015) also confirmed the protective effects of probiotics and synbiotics consumption before ab- dominal surgery, which reduced infective complications such as urinary tract infections, pneumonia and sepsis ${ }^{15,20-24}$. Liu et al. recommended a diet that included bacteria of different strains, and this diet was more useful than single-strain diets for surgical infections and other infections ${ }^{22}$.

Consoli et al. (2016) studied about probiotic (Saccharomyces boulardii) consumption in patients with colon surgery and the levels of inflammatory cytokines. They found that in the study group, the levels of mucosal IL-1 beta, IL-10 and IL- 23 were lower and probiotic administration before surgery had no effect on infective complications, duration of hospitalization and mortality ${ }^{25}$.

A numerous causes could affect the results of our study, including the strains of probiotics that were used. Perhaps, other strains of probiotics could be considered in future research. Moreover, dosage, duration and different routes of probiotic administration, such as direct administration into the jejunum, could be considered for further research. On the other hand, prophylactic antibiotics can also reduce the effect of probiotics and need to be taken into account in the future. 


\section{CONCLUSIONS}

This study showed that there is no correlation between probiotic consumption preoperatively and the decrease in complications and mortality after surgery. Study with larger groups, longer administration of probiotics or synbiotics, longer follow-up duration and other administration routes are recommended.

\section{COMPETING INTERESTS}

We don't declare under financial, general, and institutional competing interests. We had full access to all study data, take fully responsibility for the accuracy of the data analysis, and have authority over manuscript preparation and decisions to submit the manuscript for publication.

\section{AUTHORS' CONTRIBUTIONS}

Study design: Dr. F. Kakaei and Dr. M. Shahrasbi Operations: Dr. F. Kakaei

Collecting data: Dr. M. Shahrasbi and Dr. S. Taheri Data analysis: Dr. F. Kakaei and Dr. T. Asvadi Kermani

Writting the paper:Dr. T. Asvadi Kermani and Dr. K. Tarvirdizade

Editting the paper: Dr. F. Kakaei and Dr. T. Asvadi Kermani

\section{ACKNOWLEDGMENTS}

We would like to acknowledge Tabriz University of Medical Sciences for supporting.

\section{REFERENCES}

1. Tukmechi A, Bandboni M. The effect of yeast supplementation on the growth and immune system in rainbow trout (Oncorhynchus mykiss). Journal of Veterinary Research (Pulawy). 2013;68:69-78.

2. Tukmechi A, Bandboni M. Effects of Saccharomyces cerevisiae supplementation on immune response, hematological parameters, body composition and disease resistance in rainbow trout, Oncorhynchus mykiss (Walbaum, 1792). Journal of Applied Ichthyology. 2014;30:55-61. Available from: DOI: 10.1111/jai.12314.

3. Tukmechi A, Morshedi A, Delirezh N. Changes in intestinal microflora and humoral immune response following probiotic administration in rainbow trout (Oncorhynchus mykiss). Journal of Animal and Veterinary Advances. 2007;6.

4. Holzapfel WH, Haberer P, Snel J, Schillinger U, in't Veld JHH. Overview of gut flora and probiotics. International journal of food microbiology. 1998;41:85-101.

5. Rad AH. Therapeutical effects of functional probiotic, prebiotic and synbiotic foods. Tabriz: Tabriz University of Medical Sciences; 2008.

6. Goldin BR, Gorbach SL. Clinical indications for probiotics: an overview. Clinical Infectious Diseases. 2008;46:S96-S100.

7. Kaur IP, Kuhad A, Garg A, Chopra K. Probiotics: delineation of prophylactic and therapeutic benefits. Journal of Medicinal Food. 2009;12:219-35. Available from: DOI:10.1089/jmf.2007. 0544 .
8. Schrezenmeir J, de Vrese M. Probiotics, prebiotics, and synbiotics approaching a definition. The American Journal of Clinical Nutrition. 2001;73:361-4. Available from: DOI:10.1093/ ajcn/73.2.361s.

9. Saminathan M, Sieo CC, Kalavathy R, Abdullah N, Ho YW. Effect of prebiotic oligosaccharides on growth of Lactobacillus strains used as a probiotic for chickens. African Journal of Microbiological Research. 2011;5:57-64.

10. Macpherson AJ, Uhr T. Induction of protective IgA by intestinal dendritic cells carrying commensal bacteria. Science. 2004;303:1662-5. Available from: DOI:10.1126/science. 1091334.

11. Isolauri E, Sutas Y, Kankaanpaa P, Arvilommi H, Salminen S. Probiotics: effects on immunity. The American Journal of Clinical Nutrition. 2001;73:444-50. Available from: DOI:10.1093/ ajcn/73.2.444s.

12. Hotel ACP, Cordoba A. Health and nutritional properties of probiotics in food including powder milk with live lactic acid bacteria. Prevention. 2001;5:1-10.

13. Liu ZH, Huang MJ, Zhang XW, Wang L, Huang NQ, Peng $\mathrm{H}$. The effects of perioperative probiotic treatment on serum zonulin concentration and subsequent postoperative infectious complications after colorectal cancer surgery: a doublecenter and double-blind randomized clinical trial. The American Journal of Clinical Nutrition. 2013;97:117-26. Available from: DOI:10.3945/ajen.112.040949.

14. Aisu N, Tanimura S, Yamashita Y, Yamashita K, Maki K, Yoshida Y. Impact of perioperative probiotic treatment for surgical site infections in patients with colorectal cancer. Experimental and Therapeutic Medicine. 2015;10:966-72. Available from: DOI:10.3892/etm.2015.2640.

15. Liu Z, Qin H, Yang Z, Xia Y, Liu W, Yang J. Randomised clinical trial: the effects of perioperative probiotic treatment on barrier function and post-operative infectious complications in colorectal cancer surgery-a double-blind study. Alimentary Pharmacology \& Therapeutics. 2011;33:50-63. Available from: DOI:10.1111/j.1365-2036.2010.04492.x.

16. McNaught CE, Woodcock NP, MacFie J, Mitchell CJ. A prospective randomised study of the probiotic Lactobacillus plantarum $299 \mathrm{~V}$ on indices of gut barrier function in elective surgical patients. Gut. 2002;51:827-31. Available from: DOI: 10.1136/gut.51.6.827.

17. Anderson $A D, M c N a u g h t ~ C E$, Jain PK. Randomised clinical trial of synbiotic therapy in elective surgical patients. Gut 2004:53:241-5. Available from: DOI:10.1136/gut.2003.024620.

18. Sugawara G, Nagino M, Nishio H, Ebata T, Takagi K, Asahara T. Perioperative synbiotic treatment to prevent postoperative infectious complications in biliary cancer surgery: a randomized controlled trial. Annals of Surgery. 2006;244:706-14. Available from: DOI:10.1097/01.sla.0000219039.20924.88.

19. Rayes N, Seehofer D, Theruvath T, Mogl M, Langrehr JM, Nussler NC. Effect of enteral nutrition and synbiotics on bacterial infection rates after pylorus-preserving pancreatoduodenec tomy: a randomized, double-blind trial. Annals of Surgery. 2007;246:36. Available from: DOI:10.1097/01.sla.0000259442. 78947.19.

20. Liu Z, Li C, Huang M, Tong C, Zhang X, Wang L. Positive regulatory effects of perioperative probiotic treatment on postoperative liver complications after colorectal liver metastases surgery: a double-center and double-blind randomized clinical trial. BMC Gastroenterology. 2015;15:34. Available from: DOI:10.1186/s12876-015-0260-z.

21. Arumugam S, Lau CS, Chamberlain RS. Probiotics and synbiotics decrease postoperative sepsis in elective gastrointestinal surgical patients: a meta-analysis. Journal of Gastrointestinal Surgery. 2016;20:1123-31. Available from: DOI:10.1007/ s11605-016-3142-y.

22. Liu PC, Yan YK, Ma YJ, Wang XW, Geng J, Wang MC. Probiotics reduce postoperative infections in patients undergoing colorectal surgery: a systematic review and meta-analysis. Gastroenterology Research and Practice. 2017;2017:2017. Available from: Doi:10.1155/2017/6029075. 
23. Yang Z, Wu Q, Liu Y, Fan D. Effect of Perioperative Probiotics and Synbiotics on Postoperative Infections After Gastrointestinal Surgery: A Systematic Review With Meta-Analysis. Journal of Parenteral and Enteral Nutrition. 2017;41:10511062.

24. Kasatpibal N, Whitney JD, Saokaew S, Kengkla K, Heitkemper MM, Apisarnthanarak A. Effectiveness of Probiotic, Prebiotic, and Synbiotic Therapies in Reducing Postoperative Complications: A Systematic Review and Network Meta-analysis.
Clinical Infectious Diseases. 2017;64:153-60. Available from: DOI:10.1093/cid/cix114.

25. Consoli MLD, da Silva RS, Nicoli JR, Bruña-Romero O, da Silva RG, de Vasconcelos Generoso S, et al. Randomized clinical trial: impact of oral administration of Saccharomyces boulardii on gene expression of intestinal cytokines in patients undergoing colon resection. Journal of Parenteral and Enteral Nutrition. 2016;40:1114-1121. 